\title{
Intravitreal Anti-Vascular Endothelial Growth Factor for Macular Edema due to Complex Retinal Arterial Macroaneurysms
}

\author{
Caroline Bormann $^{a, b}$ Jens Heichel ${ }^{b} \quad$ Ute Hammer $^{a} \quad$ Anke Habermann ${ }^{a}$ \\ Thomas Hammer ${ }^{a, b}$ \\ a Augenzentrum "Frohe Zukunft", Halle (Saale), Germany; ${ }^{b}$ Department of Ophthalmology, \\ University Hospital of Martin Luther University Halle-Wittenberg, Halle (Saale), Germany
}

\section{Keywords}

Aflibercept · Laser photocoagulation · Macular edema $\cdot$ Ranibizumab · Retinal arterial macroaneurysm $\cdot$ Anti-vascular endothelial growth factor

\begin{abstract}
Introduction: Complex retinal arterial macroaneurysms (RAM) are often accompanied by hemorrhage and/or affect the macula. We evaluated the effect of intravitreal anti-vascular endothelial growth factor (anti-VEGF) therapy using ranibizumab or aflibercept with or without laser photocoagulation in the treatment of macular edema due to RAM. Methods: A case report of two patients with secondary macular edema caused by RAM is presented. The first case was a 76-year-old female treated with two 0.5 -mg injections of ranibizumab and additional focal laser photocoagulation. This patient presented a solely intraretinal exudation. The second patient was a 96-year-old female, who received one $2.0-\mathrm{mg}$ injection of aflibercept. She showed sub- and intraretinal edema. We documented the clinical courses of these patients based on fundus photography, fluorescein angiography, and spectral-domain optical coherence tomography. Patients were followed-up for 12 months. Results: Patients were treated successfully using anti-VEGF therapy (ranibizumab or aflibercept) with or without laser photocoagulation. In both cases, we observed a complete regression of the macular edema and an increase in visual acuity. Conclusion: RAM can manifest with heterogeneous
\end{abstract}


findings. Intravitreal anti-VEGF therapy with or without laser photocoagulation may be an effective treatment option in cases of macular edema due to RAM. Aflibercept and ranibizumab seem to be a potent anti-VEGF therapy for RAM. Individualized patient care is needed.

\section{Introduction}

Retinal arterial macroaneurysms (RAMs) are unilateral, spindle-shaped dilatations of the large retinal arterioles. RAMs are typically located across the superior temporal vessel arch $(50 \%)$ or at the inferior temporal arcade (45\%) [1]. Reports have suggested that older women and those with a history of hypertension represent the majority of cases [2]. Up to $10 \%$ of RAMs are bilateral or develop in multiple locations [3].

Based on the clinical course, cases of RAM can be described as either simple or complex [4]. Simple RAMs mainly consist of a vascular ectasia and are not associated with any retinal changes; this type of lesion often is an incidental finding and regresses without treatment. Complex RAMs are frequently accompanied by preretinal, retinal, subretinal, or vitreous hemorrhage or by macular edema, either of which can decrease visual acuity. The ideal treatment for complex RAMs has been the subject of controversy. Possible therapy options include laser photocoagulation, anti-vascular endothelial growth factor (VEGF) injections, Nd:YAG laser hyaloidotomy, or one of several different surgical interventions combined with pars plana vitrectomy (e.g., intravitreal administration of gas or recombinant tissue plasminogen activator) $[4,5]$.

Recently, research has suggested that VEGF may be involved in the pathology of complex RAM development. Successful treatment of these lesions with intravitreal anti-VEGF therapy has already been shown in several case reports and studies [6-9]. However, the pathogenesis has not been fully clarified. Here we present two cases of patients with RAMs who were treated with anti-VEGF therapy with or without concurrent laser photocoagulation.

\section{Case Report}

Two patients, a 76-year-old woman (patient 1) and a 96-year-old woman (patient 2), presented to our clinic with unilateral indolent vision loss and metamorphopsia of the left eye. Both women had a medical history of hypertension and had undergone cataract surgery in the left eye without complications.

\section{Patient 1}

History and Findings: At the first examination, the best-corrected visual acuity (BCVA) was 20/50 in her right eye (OD) and 20/70 in her left eye (OS). Intraocular pressure and slitlamp biomicroscopy of the anterior segment were unremarkable. On the fundus exam, we visualized a pre- and intraretinal hemorrhage surrounded by intraretinal edema and hard exudates located at the inferior temporal vessel arch. Fluorescein angiography (FA) and optical coherence tomography (SD-OCT) confirmed the diagnosis of complex RAMs with secondary macular edema.

Therapy and Clinical Course: The patient received a first intravitreal injection of ranibizumab $(0.5 \mathrm{mg})$ in her left eye. Four weeks later, we observed a significant but incomplete 
reduction in the edema. However, after 4 weeks, visual acuity got worse (BCVA 20/100). Therefore, we decided to treat her with a second intravitreal injection combined with focal laser photocoagulation. After an additional ranibizumab injection $(0.5 \mathrm{mg})$ and a laser treatment around the aneurysm, her visual acuity increased to 20/25 and remained stable during a follow-up period of 12 months. There was a complete regression of the macular edema and thrombosis of the RAM (Fig. 1a-d).

\section{Patient 2}

History and Findings: BCVA was 20/30 OD and 20/200 OS at the first consultation. Intraocular pressure and slit-lamp biomicroscopy of the anterior segment were regular. The fundus exam revealed a preretinal hemorrhage above the fovea and with surrounding edema. The right eye of each patient did not show any pathology. FA and SD-OCT confirmed the diagnosis of complex RAMs with secondary macular edema and subretinal fluid.

Therapy and Clinical Course: The patient was treated with one intravitreal injection of aflibercept $(2.0 \mathrm{mg})$. After 4 weeks, she had a complete resolution of the macular edema, which remained stable during the follow-up period of 12 months as well. Her BCVA increased after 4 weeks to 20/100, after 8 weeks to 20/70, and after 12 months to 20/50 (Fig. $2 \mathrm{a}-\mathrm{c})$.

\section{Compliance with Ethics Guidelines}

All procedures were done in accordance with the ethical standards of the responsible committee on human experimentation (institutional and national) and with the Helsinki Declaration of 1964, as revised in 2013. Informed consent was obtained from all patients for being included in this retrospective analysis.

\section{Discussion}

These 2 case reports comprise the typical findings of RAM and a successful intravitreal anti-VEGF therapy. The treatment of symptomatic (complex) RAM remains challenging and a subject of debate. There are no evidence-based treatment regimens for these aneurysms. However, there has been a consensus for treatment for complications such as macular edema or macular or vitreous hemorrhage [4, 5]. For this, intravitreal injections can be considered, and there has been published data about the use of bevacizumab and ranibizumab [7, $8,10]$. Data concerning the use of aflibercept do not exist so far.

In a retrospective study of 23 eyes with symptomatic RAM, the authors compared treatment with bevacizumab (11 eyes) to observation without any treatment (12 eyes). Patients presented clinical signs of RAM consisting of sub- and intraretinal fluid, hard exudates, and intraretinal hemorrhage. In the bevacizumab group, there was a significant increase in visual acuity [11]. Pichi et al. [9] prospectively studied the use of bevacizumab in 37 cases of similar clinical findings and found that the functional and anatomical endpoints had improved significantly after bevacizumab treatment. A retrospective study of 10 eyes with RAM and macular exudates without hemorrhage did not demonstrate significant differences in anatomical or functional results after treatment with ranibizumab or bevacizumab [12].

According to published findings, FA demonstrates sharply defined hyperfluorescence at the affected retinal artery. During FA, an increasing intensity of the fluorescence can be observed. In the late phase, a leakage occurs. In case of hemorrhagic RAM, a typical blockade phenomenon is visible $[8,9,12]$. Furthermore, OCT shows an elevated retina covering a 
hyporeflective lumen with a hyperreflective wall. Intra- and subretinal fluid are other typical findings $[8,9,11,12]$.

These clinical studies allow us to conclude that anti-VEGF therapy can work in cases with symptomatic RAM, but the mechanism of action has remained unclear. However, there have been two hypotheses about the effect of this therapy. On the one hand, anti-VEGF may reduce nitrogen oxide production in the endothelium with vasoconstriction, the decrease in vascular permeability, and edema reduction. Additionally, the balance between coagulation and fibrinolysis is improved $[11,12]$.

In our patients, we were able to successfully use intravitreal anti-VEGF therapy to treat symptomatic complex RAM. Intravitreal anti-VEGF injection for RAM is an outpatient, minimally invasive treatment with a low rate of complications. A rapid involution of macular edema due to a complex RAM was observed after intravitreal anti-VEGF therapy. Although there was a minor hemorrhagic component, exudation into the macula was the major criterion to initiate treatment. As the clinical manifestation of RAM can be very heterogeneous, we studied two patients, one with only intraretinal edema and one with intra- and subretinal fluid.

Nevertheless, the natural course of RAM which undergoes spontaneous involution with time must be taken into consideration. Indications for treatment or retreatment therefore are based on signs of activity, such as progressive or ongoing exudative changes. SD-OCT and fundus exams are appropriate tools as well as FA (leakage from the RAM).

One patient showed decreased vision 4 weeks after initial anti-VEGF injection, which does not automatically mean that retreatment is indicated. Function initially may drop as a result of accumulation of lipid exudates in the fovea even though treatment (either with focal laser or anti-VEGF) was successful in terms of stopping leakage and improving macular edema. These findings hint on an increased phagocytotic activity in the retinal tissue. With time, those hard exudates could resolve and vision gradually would improve. Nevertheless, we decided to treat her with a second intravitreal injection according to the therapy regimen used for neovascular age-related macular degeneration.

Our case report was limited because no patient was treated entirely with laser photocoagulation and different anti-VEGF drugs were used. Moreover, to the best of our knowledge, this case report is the first about the effectiveness of aflibercept in RAM. A prospective, controlled clinical study could clarify the different therapeutic options for the treatment of complex RAMs. Also the use of aflibercept should be examined in further studies.

\section{Statement of Ethics}

All procedures were in accordance with the ethical standards of the responsible committee on human experimentation (institutional and national) and with the Helsinki Declaration of 1964, as revised in 2013. Informed consent was obtained from all patients for being included in the retrospective analysis.

\section{Disclosure Statement}

Caroline Bormann, Jens Heichel, Ute Hammer, Anke Habermann, and Thomas Hammer have nothing to declare. No funding or sponsorship was received for this study or publica- 
tion of this article. All named authors meet the International Committee of Medical Journal Editors (ICMJE) criteria for authorship for this case report, take responsibility for the integrity of the work as a whole, and have given final approval to the version to be published.

\section{References}

1 Moosavi RA, Fong KC, Chopdar A: Retinal artery macroaneurysms: clinical and fluorescein angiographic features in 34 patients. Eye (Lond) 2006;20:1011-1020.

-2 Panton RW, Goldberg MF, Farber MD: Retinal arterial macroaneurysms: risk factors and natural history. Br J Ophthalmol 1990;74:595-600.

-3 Rabb MF, Gagliano DA, Teske MP: Retinal arterial macroaneruysms. Surv Ophthalmol 1988;33:73-76.
4 Bopp S, Joussen AM: Retinal arterial macroaneurysms (RAM) - pathology, differential diagnoses and therapy (in German). Klin Monbl Augenheilkd 2014;231:890-900.

5 Schüler A: Retinales arterielles Makroaneurysma und retinales kavernöses Hämangiom. In: Heimann $\mathrm{H}$, Kellner U (eds): Altlas des Augenhintergrundes. Stuttgart: Georg Thieme Verlag KG, 2010, pp 140-141.

-6 Wenkstern AR, Petersen H: Intravitreal ranibizumab in retinal macroaneurysm. Graefes Arch Clin Exp Ophthalmol 2010;248:1167-1170.

7 Tsakpinis D, Nasr MB, Tranos P, et al: The use of bevacizumab in a multilevel retinal hemorrhage secondary to retinal macroaneurysm: a 39-month follow-up case report. Clin Ophthalmol 2011;5:1475-1477.

8 Menezes C, Carvalho R, Teixeira C, et al: Foveal exudative macroaneurysm treated with intravitreal ranibizumab. Case Rep Ophthalmol 2015;6:170-175.

9 Pichi F, Morara M, Torrazza C, et al: Intravitreal bevacizumab for macular complications from retinal arterial macroaneurysms. Am J Ophthalmol 2013;155:287-294.

$\$ 10$ Leung EH, Reddy A, Vedula A, et al: Serial bevacizumab injections and laser photocoagulation for macular edema associated with a retinal artery macroaneurysm. Clin Ophthalmol 2015;9:601-609.

11 Cho HJ, Rhee TK, Kim HS, et al: Intravitreal bevacizumab for symptomatic retinal arterial macroaneurysm. Am J Ophthalmol 2013;155:898-904.

-12 Zweifel SA, Tönz MS, Pfenniger L, et al: Intravitreal anti-VEGF therapy for retinal macroaneurysm. Klin Monbl Augenheilkd 2013;230:392-395.

C.B. and J.H. contributed equally to this work 


\section{Case Reports in Ophthalmology}
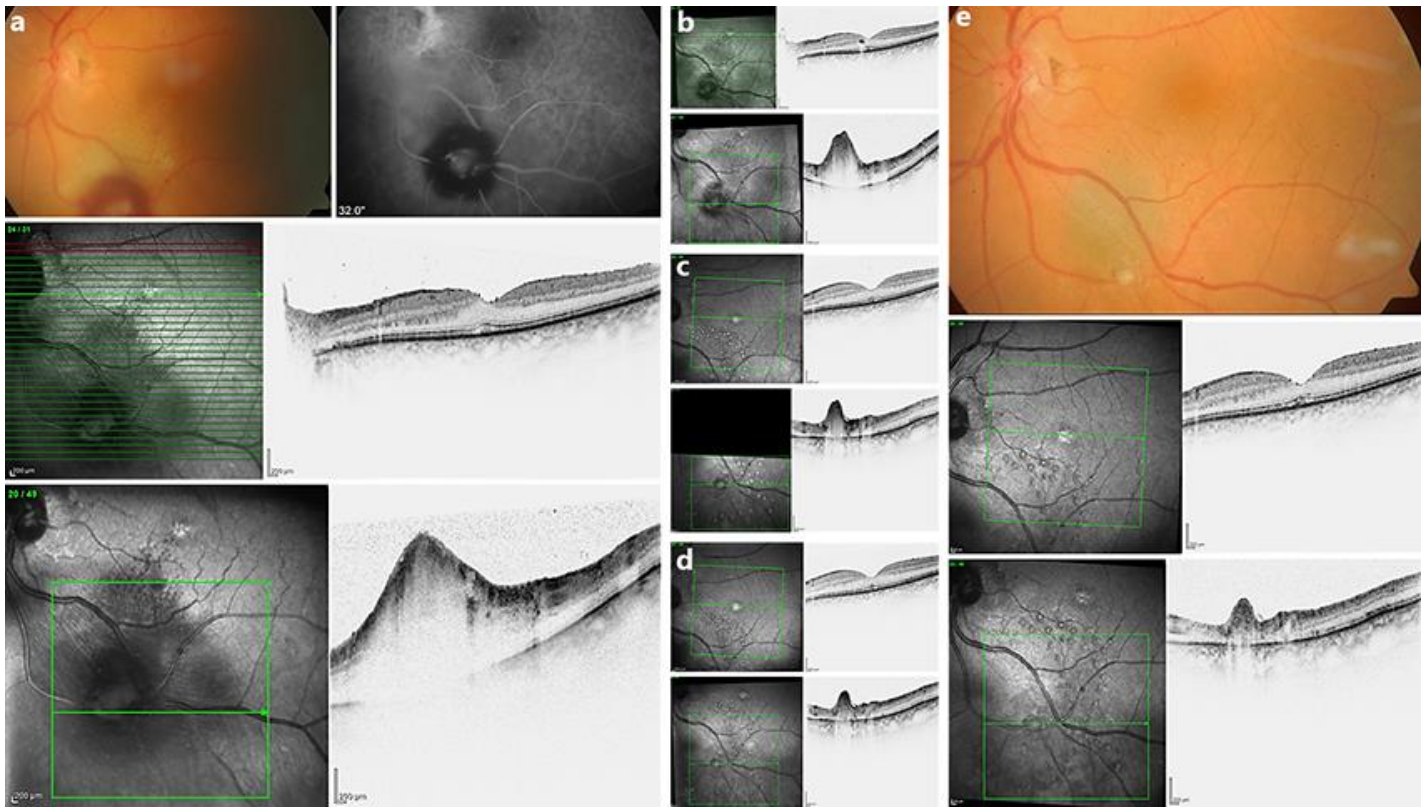

Fig. 1. Findings and clinical course of patient 1 (left eye). a Findings at first presentation (best-corrected visual acuity, BCVA, 20/70). Upper row: color photograph (left) shows a retinal arterial macroaneurysm (RAM) located at the inferior temporal vessel arch with pre- and intraretinal hemorrhage, surrounding edema, and hard exudates. Fluorescein angiography (right) demonstrates a sharply defined hyperfluorescent RAM with surrounding blockage caused by hemorrhage. Middle row: infrared foto (IR) (left) and spectral-domain optical coherence tomography (SD-OCT) (right) of the fovea without edema. Lower row: IR (left) and SD-OCT (right) of the RAM showing intraretinal fluid and an elevation of the retina. b One month after the first intravitreal injection of $0.5 \mathrm{mg}$ of ranibizumab (BCVA 20/100). IR (left) and SD-OCT (right) reveal stable foveal findings without exudation and a reduction of retinal edema in the region of the RAM (upper row: foveal scan; lower row: RAM). c One month after a second intravitreal injection of $0.5 \mathrm{mg}$ of ranibizumab and focal laser photocoagulation (BCVA 20/30). IR (left) and SD-OCT (right) give no evidence for subfoveal edema and ongoing fluid reduction near the RAM (upper row: foveal scan; lower row: RAM). d Four months after c (BCVA 20/25). IR (left) and SD-OCT (right) show a constant foveal configuration and further regression of RAM (upper row: foveal scan; lower row: RAM). e Twelve months after second intravitreal injection of $0.5 \mathrm{mg}$ of ranibizumab and focal laser photocoagulation (BCVA 20/25). Color photograph (upper row), IR (middle and lower row, left), and SD-OCT (middle and lower row, right) show a complete obliteration of RAM and resolution of the pre- and intraretinal hemorrhage as well as retinal edema. Additionally, a nearly located choroidal nevus is visible. 


\section{Case Reports in Ophthalmology}
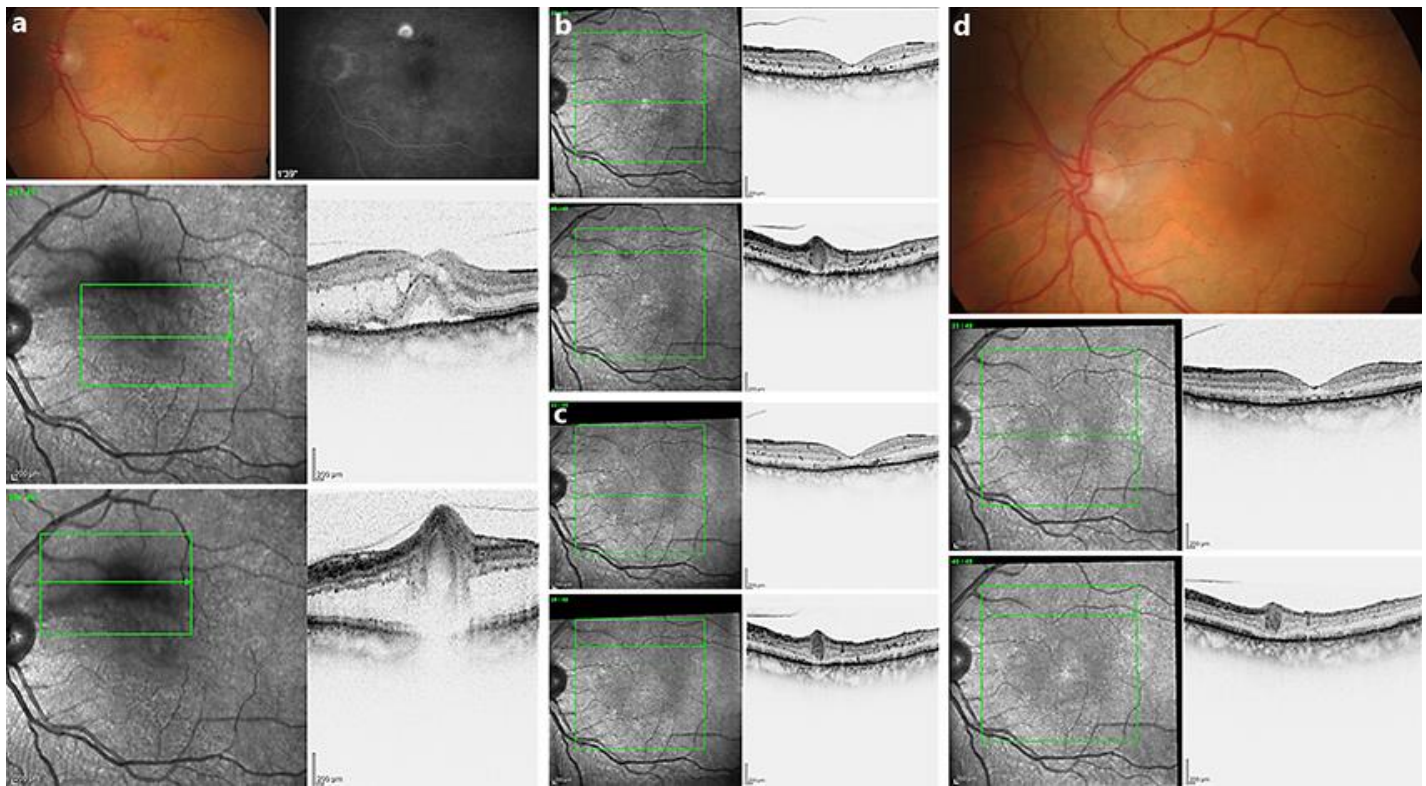

Fig. 2. Findings and clinical course of patient 2 (left eye). a Findings at first presentation (best-corrected visual acuity, BCVA, 20/200). Upper row: color photograph (left) presents a RAM located above the fovea with surrounding edema and pre- and intraretinal hemorrhage. Fluorescein angiography (right) signalizes a sharply defined hyperfluorescence. Middle row: infrared foto (IR) (left) and spectral-domain optical coherence tomography (SD-OCT) (right) of the fovea indicate intra- and subretinal fluid. Lower row: IR (left) and SD-OCT (right) of the RAM illustrate significant intraretinal fluid. b One month after intravitreal injection of $2.0 \mathrm{mg}$ of aflibercept (BCVA 20/100). c Three months after b (BCVA 20/70). Complete regression of sub- and intraretinal fluid as well as involution of RAM is confirmed (IR left, SD-OCT right; upper row: foveal scan, lower row: RAM). $\mathbf{d}$ Twelve months after intravitreal injection of $2.0 \mathrm{mg}$ of aflibercept (BCVA 20/50). Color photograph (upper row), IR (middle and lower row, left), and SD-OCT (middle and lower row, right) indicate a complete obliteration of RAM. Signs of relapse of pre- and intraretinal hemorrhage as well as sub- and intraretinal fluid are not present. 